\title{
$X$-ray diffractometer stage for in situ structural analysis of thin films
}

\author{
R.W. Johnson and W. L. Johnson \\ W. M. Keck Laboratory of Engineering Materiais, California Institute of Technology, Pasadena, \\ California 91125
}

(Received 7 Juiy 1988; accepted for publication 19 August 1988)

\begin{abstract}
A theta-two theta $\mathrm{x}$-ray diffractometer stage has been developed for in situ structural characterization of thin-film samples. This stage integrates an uttrahigh vacuum dc ion-beam thin-film sample preparation chamber with the Siemens D500 $\mathrm{x}$-ray diffractometer. In vacuo sample translation and manipulation is provided. The stage incorporates resistive heating to 900 $\mathrm{K}$ and liquid nitrogen cooling to $150 \mathrm{~K}$. The sample theta rotation is transmitted into the vacuum chamber by a rotary feedthrough. X rays enter the vacuum chamber through a beryllium window with allowed reflection angles from 0 to $+168^{\circ}$ two-theta.
\end{abstract}

\section{INTRODUCTION}

Recently, there has been much interest in our laboratory and elsewhere in the study of interdiffusion-induced amorphization reactions in thin-film multilayer metal samples. ${ }^{1}$ These reactions rely on a suppression of nucleation (and so nucleation sites) of crystalline equilibrium phases and are highly sensitive to the presence of oxygen and other impurities. Candidates for these reactions among the rare-earth series, therefore, require a special materials handling capability. With this in mind and the wish to perform a wide range of structural analyses of materials under the cleanest possibie conditions, we have integrated the Siemens D500 theta-two theta $x$-ray diffractometer to an ultrahigh vacuum (UHV) dc ion-beam sample deposition chamber. This $x$-ray diffaction stage allows in situ structural characterization of phases formed during an annealing sequence by both high- and lowangle $\mathrm{x}$-ray diffraction measurements. The $\mathrm{x}$-ray diffraction system is functionally decoupled from the main UHV sample preparation chamber; it allows an easy transfer of samples originating both from within and external to the main UHV preparation chamber onto the $x$-ray stage. Integration was designed to utilize commercially available components as much as possible; this system and coupling scheme may be readily adaptable to other experimental configurations.

Our choice of the theta-two theta diffraction geometry was motivated by its suitability for quantitative, high-resolution, small- and high-angle $x$-ray scattering experiments, ease of operation, and the availability of commercial systems with this geometry. There have been several previous reports of the integration of $\mathrm{x}$-ray diffraction techniques with an UHV capability. ${ }^{2}$ The primary focus of these efforts was the study of thin-film surfaces by grazing incidence $x$-ray scattering. These instruments, that included four rotational degrees of freedom for the scattering of $x$ rays with respect to the sample, were designed primarily for use at synchrotron $\mathrm{x}$-ray sources. The aims of the present work are complementary. The instrument described in this paper provides a general purpose in situ $\mathrm{x}$-ray scattering capability for routine laboratory use. High-angle scattering experiments on amorphous or polycrystalline thin-film samples and small-angle scattering experiments on thin-film modulated structures comprise the primary intended applications for this instrument.

A schematic of the main UHV sample preparation chamber is shown in Fig. 1. This chamber was engineered and manufactured by the UHV Instruments, Inc. ${ }^{3}$ The chamber is in the form of a hemisphere with a 6 in. radius. An ultimate pressure of $10^{-9}$ Torr is achieved by a $1500 \ell / \mathrm{s}$ He refirigeration cryopump. Two $3-\mathrm{cm}$ Commonwealth Scientific Corp. ion guns provide a variably focused beam with energy from $20 \mathrm{eV}$ to $1 \mathrm{keV}$. These two guns can be mounted in a cosputtering or in an ion-assisted-sputtering configuration. Sputtering target selection and substrate shuttering are controlled by programmabie relays initiated by the sample deposition monitor. The temperature controlled ( -150 to $900 \mathrm{~K}$ ) substrate stage continuously rotates during deposition to ensure lateral uniformity of the sample. Substrates and targets are transferred between the main UHV chamber and the sample interlock chamber by a magnetically coupled manipulator arm. This system is standardized for use of 2 in.-diam sputtering targets and substrates. A more complete description of this system will be contained in forthcoming publications.

\section{TECHNIQUE AND APPARATUS}

The basic geometry of the theta two-theta diffractometer is shown in Fig. 2. Samples in the form of a flat plate are supported at the center of the diffractometer circle. A diverging line source of $\mathrm{x}$ rays is collimated by aperture slits. When the Bragg condition $2 d \sin \theta=n \lambda$ for diffraction from the sample is satisfied, $x$ rays are reflected. Focusing of the reflected $x$ rays is ensured if the angle of incidence on and reflection from the sample are equal. Rotation of the sample through theta degrees is coupled to rotation of the detector through two theta degrees to ensure this condition. $X$ rays reflected from the sample are collimated by detector slits, pass through a $K_{\beta}$ filter to remove the $K_{\beta}$ component of the $\mathrm{x}$-ray intensity, and are registered by a detector.

This work incorporates the commercially available Siemens D500 PCAT. ${ }^{4}$ A Kristalloflex $710 \mathrm{H}$ generator $(2700$ $\mathrm{W}, 5-60 \mathrm{~mA}$, and $20-55 \mathrm{kV}$ continuously adjustable) powers a type FK 60-04, 1500-W, Cu-target $x$-ray tube. A line 


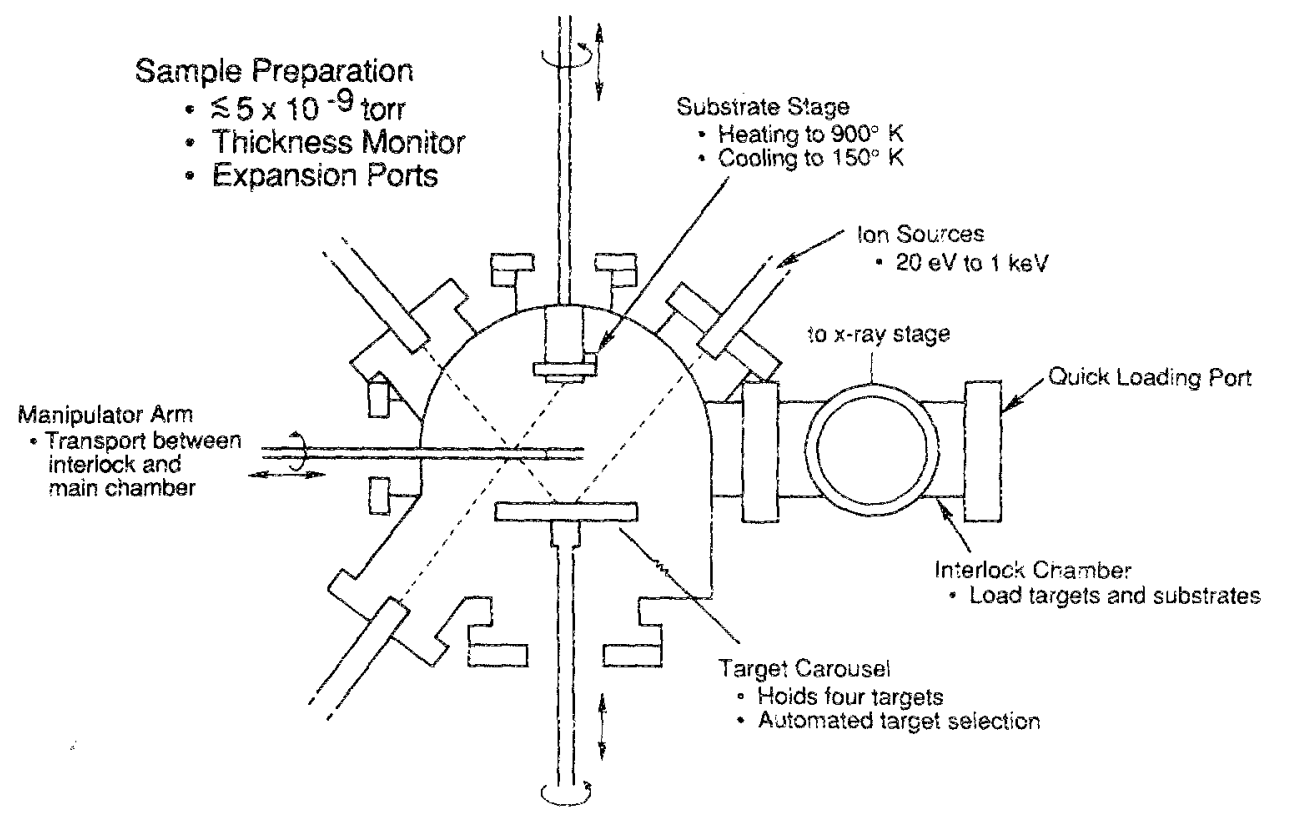

FIG. 1. Schematic view of the UHV de ionbeam sample preparation chamber. The $\mathrm{x}$-ray diffraction chamber connects to the interlock chamber and extends normal to the plane of this figure.

source of $x$ rays with optical focal area of $8 \times 0.04 \mathrm{~mm}^{2}$ is generated. The radius of the diffractometer circle is $250 \mathrm{~mm}$. The angular range of theta (sample rotation) is $360^{\circ}$ and that of two-theta (detector rotation) is $0-168^{\circ}$. Independent drives are provided for the theta and the two-theta rotational degrees of freedom. The reproducibility of the angular settings is $<0.001^{\circ}$. Plug-in aperture slits define beam divergences of $0.1,0.3,1$, and $3^{\circ}$. Plug-in detector slits allow collection angles of $0.018,0.05,0.15$, and $0.6^{\circ}$. Collimater (Soller) slits are located between the two aperture slits (angular aperture $=2.4^{\circ}$ ) and between the two detector slits (angular aperture $=2^{\circ}$ ). A Ni $K_{\beta}$ filter is located between the detector slit and the detector. A NaI (T1) scintillation counter is used to detect $x$ rays scattered from the thin-film sample. Data acquisition and collection are microprocesso controlled by the DACO MP. The DACO MP is interfaced to an IBM PC AT for keyboard control of the experimental parameters and for post-experiment data manipulation and analysis. Plotting of experimental $x$-ray curves is performed by an IBM Color Jetprinter.

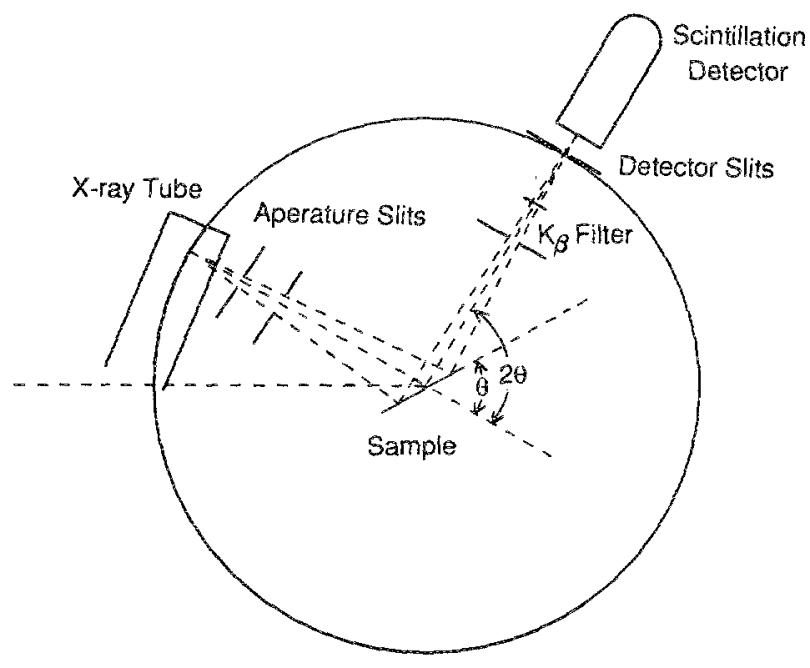

FIG. 2. The basic geometry of the theta-two theta diffractometer.
An overall view of the $x$-ray diffraction chamber is shown in Fig. 3. This chamber was manufactured by the UHV Instruments, Inc. ${ }^{3}$ A typical operating vacuum of $10^{-7}$ Torr is achieved with a $145-\ell / \mathrm{s}$ turbomolecular pump backed by a $0.7-\ell / \mathrm{s}$ rotary vane mechanical pump. The $x-$ ray chamber connects to the sample interlock chamber by a flexible stainless-steel bellows and vibration-isolation bellows support. These latter serve to vibrationally decouple the main chamber with its cryogenic and turbomolecular pumping stations from the $x$-ray stage. Samples, originating from the main preparation chamber or externally to the vacuum system, are introduced to the sample interlock chamber. Gate valves separate this interlock chamber from both the $\mathrm{x}$-ray and main chambers, allowing sample introduction with continuous evacuation of these two chambers.

Integration to the diffractometer is accomplished with a direct replacement of the theta plate standard to the Siemens D500. The theta-rotational degree of freedom is transferred into the $x$-ray stage vacuum chamber by a "ferrofluidic" rotating seal that bolts onto the theta plate with an 0 -ring seal. The exterior portion of the "ferrofuidic" seal remains stationary with the vacuum system while the interior portion rotates with the theta rotation of the sample.

$X$ rays enter the vacuum chamber through a curved 0.1 mm-thick and 20-mm-wide Be window obtained through the Edmund Beuhler Co. This window extends over $208^{\circ}$, allowing $95 \%$ transmission for $\mathrm{Cu} K_{\alpha}$ x rays scattered from both small and high angles. A cooling water channel surrounds the Be window. Extender arms, constructed on the design of those commercially available for the Siemens Eulerian cradle attachment, ${ }^{4}$ position the $\mathrm{X}$-ray tube and detector to the Be window.

An expanded schematic of the $x$-ray stage itself is shown in Fig. 4. Attachment of the stage to the theta plate is accomplished with three 0.035 -in--o.d. music wires that have been threaded through precisely matched, single-bore ceramic spacers. These music wires are welded to a stainless-steel ring clasp on the theta-plate extension and to a sliding ring 


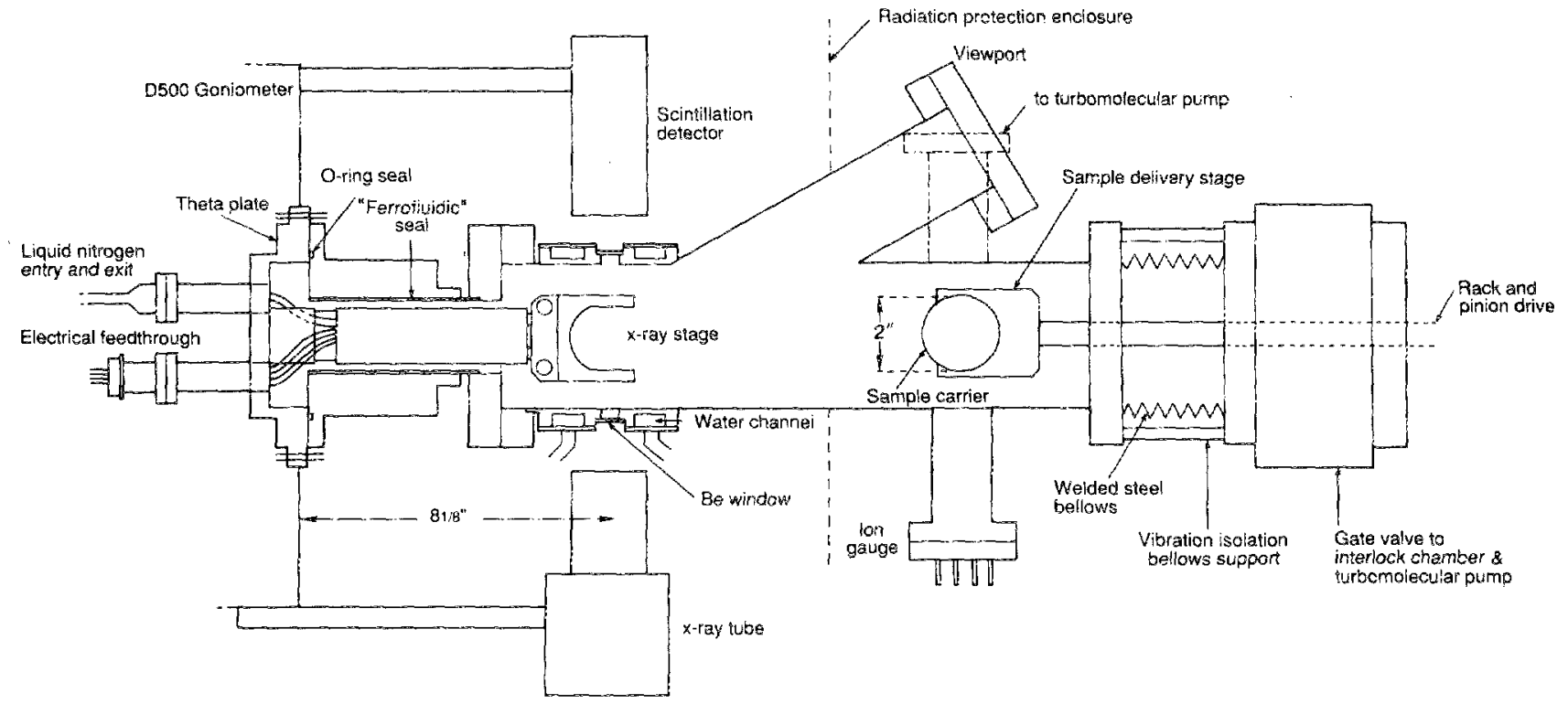

Fig. 3. Horizontal-plane view of the $\mathrm{x}$-ray diffraction chamber and stage.

abutting a stationary ring clasp on the liquid-nitrogen well of the $x$-ray stage. These wires are held under high tension by three set screws on the sliding ring. Additional support is provided by the two insulated and tightly anchored $\frac{1}{8}$-in.-o.d. stainless-steel liquid-nitrogen entry and exit lines. This design was motivated by the competing demands for thermal isolation and for structural integrity.

A liquid-nitrogen well in the form of a cylinder with a hollow bore provides sample cooling. The $\mathrm{x}$-ray stage sample fork is attached firmly to the liquid-nitrogen well with a copper ring clasp. The $\mathrm{x}$-ray stage is heated resistively with two 40-W heating cartridges. Heat transfer between the $x$-ray stage fork and the liquid-nitrogen well has been designed to allow a working temperature range from 150 to 900 $\mathrm{K}$.

The sample is mounted on an $O F H$ copper sample carrier (Fig. 4) that is transferred to the X-ray stage with a 30in.-stroke rack-and-pinion drive. ${ }^{3}$ The fork of the $x$-ray stage fits into the upper slot and the fork of the rack-and-pinion drive fits into the lower slot of the copper sample carrier. The sample carrier is spring loaded onto the $x$-ray stage fork. Removal of the sample carrier from the $\mathrm{x}$-ray stage is accom- plished by raising the fork of the rack-and-pinion drive to compress the spring clips and withdrawing the sample carrier. A glass viewport allows visual observation of the $x$-ray sample stage during sample loading and unloading.

Two additional $2 \frac{3}{4}$-in. and three additional $1 \frac{1}{3}$-in. vacuum flanges (not shown in Fig. 3) are available on the $x$-ray stage vacuum chamber for future analytic instrumentation.

The window of the as-delivered Siemens D500 radiation enclosure was removed and cut to a pattern allowing entry of the $x$-ray vacuum chamber extension. This window was then reinstalled with molded lead joints on the radiation enclosure. This window supports a primary load of the $x$-ray stage vacuum chamber.

\section{ALIGNMENT}

Alignment of the $x$-ray tube and incident and reflected beam apertures proceeds as described in the Siemens D500 operating instructions. ${ }^{4}$ An adjusting glass slit consisting of a glass plate with etched channel cemented onto a Si wafer is used in these measurements. The offset of the true zero of the theta angle from the measured zero is determined using the
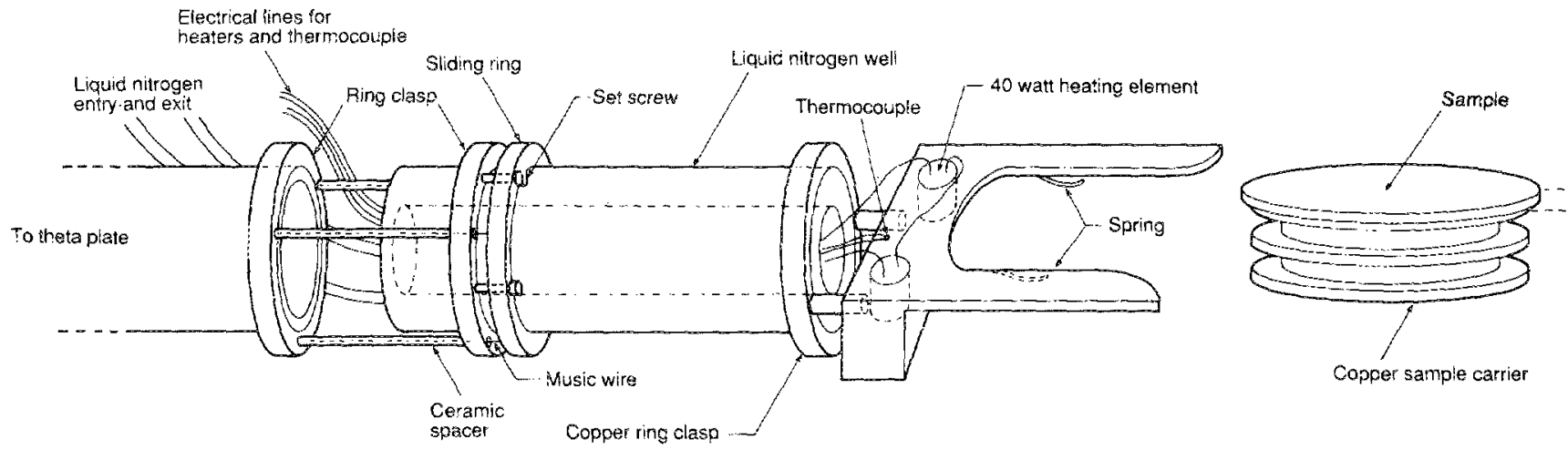

FIG. 4. Schematic view of the $\mathrm{x}$-ray sample stage. Also shown is the copper sample carrier. 
adjusting glass slit. This offset enters as a parameter in the experiment-setup software.

Positioning of the sample plane with the center of the diffractometer circle is the single alignment unique to this stage. This plane was determined from the as-delivered D500 and alignment tools were constructed to reproduce this plane. The distance from the top surface of the $x$-ray stage fork to the top surface of the sample carrier was chosen so that a $\mathrm{Si}$ water affixed to the sample carrier would be correctly positioned.

\section{PERFORMANCE}

Figure 5 illustrates a high-angle diffraction test scan on 300 mesh cubic Fd $3 \mathrm{~m}$ Si powder that was foated to near the proper thickness with acetone. The chamber was at atmospheric pressure during this scan in order to avoid fuffing of the powder during evacuation. Data were collected using a $1^{\circ}$ divergent slit and a $0.15^{\circ}$ receiving slit in steps of $0.1^{\circ}$ with a $1-s$ counting time per step. The observed positions of the Bragg reflections agree with published values. The $K_{c t_{t, 2}}$ angular splitting $d 2 \theta=2 \tan \theta d \lambda / \lambda$, where $d \lambda$ is the difference in these respective wavelengths, is evident for the backscattering angles. A slight amount of preferred orientation is observed in this sample. The inset shows a higher resolution scan taken on the $(111\rangle$ peak of $\mathrm{Si}$. A $0.3^{\circ}$ divergent slit and a $0.05^{\circ}$ receiving slit were used. Data were taken in steps of $0.005^{\circ}$ with a 1 -s counting time per step. The $K_{\alpha_{1,2}}$ splitting is clearly resolved for this peak. The observed angular position of $29.38^{\circ}$ is shifted from the true value of $28.44^{\circ}$ due to a slight displacement of the sample plane with respect to the center of the diffractometer circle. The offset in twotheta due to this displacement can be expressed $d 2 \theta=2 \cos \theta s / r$, where $s$ is the sample displacement and $r$ is the diffractometer radius. A displacement $s=0.13 \mathrm{~mm}$ below the center of the diffractometer circle is indicated for this sample.

CuY multilayer samples were prepared in the UHV sample preparation chamber and analyzed by both high - and low-angle $x$-ray diffraction and by cross-sectional transmission electron microscopy. Figure 6 illustrates one typica! small-angle $x$-ray difraction scan obtained at room temperature. This sample, with total thickness of $2115 \AA$ is com-

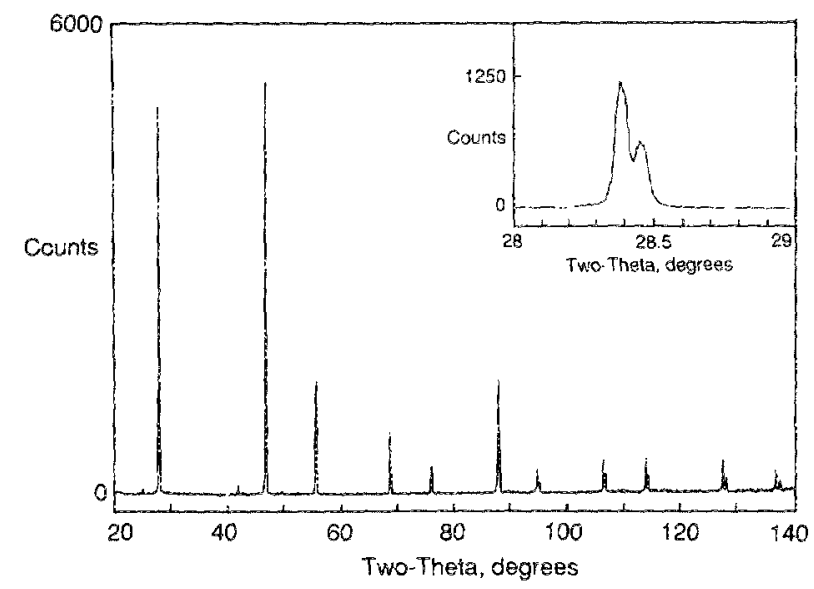

FIG. 5. High-angle test scan on a Si powder standard. Inset shows a higher resolution scan of the $\{111\}$ Si reflection.

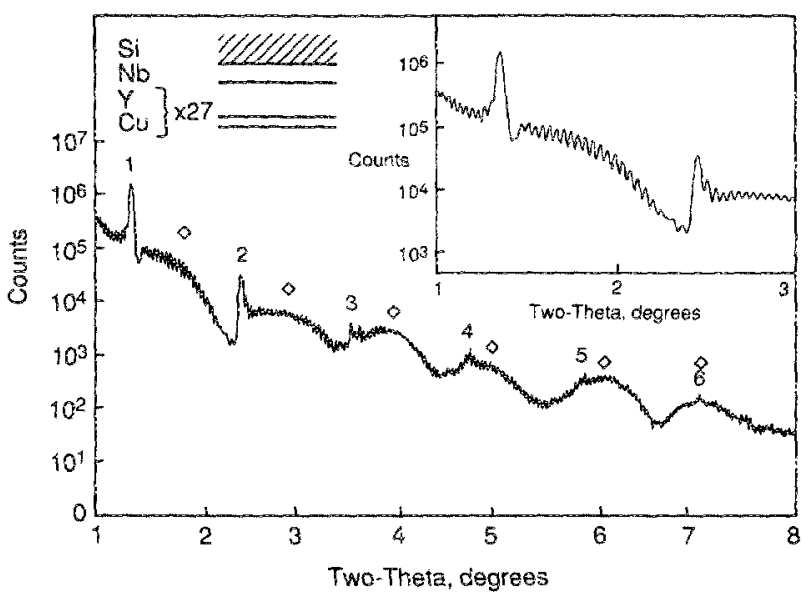

Fig. 6. Small-angle scan on a thin-film sample consisting of $90 \AA$ of $\mathrm{Nb}$ deposited on a (111) Si waifer and followed by 27 YCu bilayers, each consisting of $55 \AA Y$ and $20 \AA \mathrm{Cu}$. The orders of the small-angle satellite peaks due to the $C u Y$ composition modulation are numbered. Diamonds indicate the broad maxima due to the $\mathrm{Nb}$ underlayer. The inset shows an expanded view of the high angular-frequency oscillations that arise due to the total CuY film thickness.

posed of a $90 \mathrm{~A}$ diffusion barrier of $\mathrm{Nb}$ deposited on (111) Si and followed by 27 YCu bilayers, each consisting of $55 \AA Y$ and $20 \AA \mathrm{Cu}$. The incident beam divergence was $0.1^{\circ}$ and the collection angle was $0.018^{\circ}$. Data were collected in steps of $0.005^{\circ}$ two-theta with a counting time of $25 \mathrm{~s}$ per step. Modulations in the small-angle scattered intensity due to the total $\mathrm{CuY}$ fim thickness, the CuY composition modulation, and due to the $90 \AA \mathrm{Nb}$ layer are evident in these data. The inset shows an expanded view of the high angular-frequency oscillations that when analyzed according to procedures described by Segmülier ${ }^{6}$ provide a measure of the total $\mathrm{CuY}$ film thickness (probable error $=2 \%$ ) and the real refractive index of the sample. Small-angle satellite peaks due to the CuY composition modulation are evident to the sixth order. The broad peaks noted with diamonds in Fig. 6 are due to the $90 \AA \mathrm{Nb}$ difusion barrier. These data illustrate the highresolution capability of this instrument for small-angle $x$-ray diffraction measurements.

This x-ray diffractometer stage can be upgraded with components that are commercially available for the Siemens D500. Straightforward enhancements could include the addition of a position-sensitive detector for real-time annealing studies, the addition of incident or diffracted beam monochromators, and the use of a solid-state detector for energy dispersive work. ${ }^{4}$

\section{ACKNOWLEDGMENTS}

K. Samwer and L. Lowry provided assistance in obtaining certain components for the $x$-ray stage. We greatly appreciate the technical support of $L$. F. Johnson. One of us (R. W. J.) wishes to acknowledge the Chaim Weizmann Foundation for financial support during the course of this work.

This work has been supported by the National Science Foundation-Materials Research Groups Grant No. DMR 8421119. 
'W. L. Johnson, Mater. Sci. Eng. 97, 1 (1988); and refs. cited therein. ${ }^{2}$ E. Vlieg, A. van't Ent, A. P. DeJongh, H. Neerings, and J. F. van der Veen, Nucl. Instrum. Methods A 262, 522 (1987); P. H. Fuoss and I. K. Robinson, Nucl. Instrum. Methods 222, 171 (1984); S. Brennan and P. Eisenberger, Nucl. Instrum. Methods 222, 164 (1984).

${ }^{3}$ Ultra High Vacuum Instruments, Ine, Office Box 12, 1951 Hamburg Turnpike, Buffalo, NY 14218
${ }^{4}$ Siemens Energy and Automation, Inc., 1 Computer Drive, P.O. Box 5477 , Cherry Hill, N3 08034.

${ }^{5}$ H. P. Klug and L. E. Alexander, X-ray Diffraction Procedures for Polycrystalline and Amorphous Materials (Wiley-Interscience, New York, 1974), p. 303.

'A. Segmüller, Thin Solid Films 18, 287 (1973). 